Cite as: Suarez-Cuartin G, Molina-Molina M. Assessing quality of life of idiopathic pulmonary fibrosis patients: the INSTAGE study. Breathe 2019; 15: 144-146.

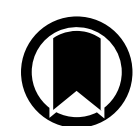

CrossMark

Journal club

\title{
Assessing quality of life of idiopathic pulmonary fibrosis patients: the INSTAGE study
}

\section{Commentary on:}

Kolb $M$, et al. Nintedanib plus sildenafil in patients with idiopathic pulmonary fibrosis. N EnglJ Med 2018; 379: 1722-1731.

\section{Context}

Idiopathic pulmonary fibrosis (IPF) is a chronic disease characterised by progressive lung interstitial fibrosis of unknown cause [1]. IPF incidence increases with older age and clinical manifestations include dry cough, exertional dyspnoea and overall progressive deterioration of patient quality of life (QOL) [1]. In the past decade, new treatment options have become available to treat IPF, such as nintedanib, an intracellular tyrosine kinase inhibitor. The INPULSIS trials evaluated the efficacy and safety of nintedanib versus placebo in 1066 IPF patients with a diffusing capacity of the lung for carbon monoxide (DLCO) of 30-79\% of the predicted value [2]. These trials showed that treatment with nintedanib slowed the rate of forced vital capacity (FVC) decline but no significant difference was observed in the St George's Respiratory Questionnaire (SGRQ) score [2].

Sildenafil is a phosphodiesterase-5 inhibitor used frequently to treat pulmonary hypertension. The sildenafil trial of exercise performance in IPF (STEP-IPF) included 180 patients with a DLCO $<35 \%$ predicted, randomised to sildenafil or placebo [3]. There was no significant difference in 6-min walk distance between groups, but stability of QOL questionnaire scores was observed in the sildenafil group while scores of the placebo group worsened [3].

Current IPF treatment guidelines do not recommend the routine use of sildenafil. Furthermore, previous clinical trials with antifibrotic medication in IPF excluded patients with severe functional impairment [4]. Therefore, investigators for the INSTAGE trial aimed to assess the potential benefits of the combined nintedanib plus sildenafil therapy for IPF patients with severe disease [5].

\section{Methods}

This was a randomised, double-blind, multicentre and parallel-group study including 13 countries. Study participants were $\geq 40$ years old, diagnosed with IPF in the previous 6 years and with a DLCO $\leq 35 \%$ of predicted value. Participants were randomly assigned in a 1:1 ratio to a treatment group of nintedanib (150 mg twice daily) plus sildenafil (20 mg three times daily) or to the nintedanib (150 mg twice daily) plus placebo (three times daily) group for 24 weeks. Echocardiographic signs suggestive of right heart dysfunction were used for stratification of randomised patients. The primary end-point was change in the SGRQ score at week 12 , which was analysed according to demographic data, presence of echocardiographic

@ERSpublications

The addition of sildenafil to nintedanib treatment does not improve outcomes to a greater extent than monotherapy with nintedanib, and it is not recommended for IPF subjects http://ow.ly/Gaq530o9kEg 
signs indicative of right heart dysfunction, FVC and DLCO percentage of predicted values, presence of emphysema, and history of previous treatment with nintedanib. Secondary end-points were: 1) change in the SGRQ score at week 24; 2) change in the University of California, San Diego, Shortness of Breath Questionnaire (UCSD-SOBQ) and the EuroQol Group 5-Dimension Self-Report Questionnaire (EQ-5D) visual-analogue scale scores at weeks 12 and 24;3) percentage of patients with serious adverse events; 4) FVC, oxygen saturation and DLCO at weeks 12 and 24; 5) change in brain natriuretic peptide (BNP) levels at week 24;6) proportion of patients with an absolute decline of $\geq 5$ percentage points of predicted value or death; 7) proportion of patients with a relative decline of $\geq 10 \%$ decline of the predicted FVC value or death; 8 ) proportion of patients with $\geq 15 \%$ decline in DLCO; 9) acute exacerbations; and 10) death from any cause, among others.

\section{Main results}

A total of 274 patients were randomised over a 14-month period, 138 of whom were assigned to the nintedanib-plus-sildenafil group and 136 to the nintedanib-plus-placebo group. Baseline patient characteristics did not differ amongst groups. Mean change from baseline SGRQ total score at week 12 was -1.28 points for the nintedanibplus-sildenafil group and -0.77 for the nintedanibplus-placebo group ( $\mathrm{p}=0.72$ ), and 0.23 points and 2.42 points, respectively, at week $24(95 \% \mathrm{Cl}$ $-5.40-1.03)$. Therefore, the primary end-point was not significant. The $p$-values for all other analyses were not reported, as they were considered to be exploratory. No significant changes in the UCSDSOBQ scores were identified at week 12 (betweengroup difference -2.94 points, $95 \% \mathrm{Cl}-7.27-1.39$ ) or at week 24 (between-group difference -2.41 points, $95 \% \mathrm{Cl}-7.39-2.58)$. Also, there were no meaningful changes in the EQ-5D visual-analogue scale scores at weeks 12 or 24 (between-group difference 3.54 points $(95 \% \mathrm{Cl}-0.02-7.09)$ and 1.50 points ( $95 \% \mathrm{Cl}-2.86-5.86)$, respectively).

The nintedanib-plus-sildenafil group had a lower risk of FVC decline of $\geq 5$ percentage points or death (31.4\% versus $50.7 \%$ of patients; hazard ratio $(H R)$ $0.56,95 \% \mathrm{Cl} 0.38-0.82)$ compared to the placebo group. Moreover, the sildenafil group had a lower percentage of patients with an FVC decline of $\geq 10 \%$ or death $(25.5 \%$ versus $36.8 \%$; $\mathrm{HR} 0.68,95 \% \mathrm{CI}$ 0.44-1.05).

No relevant differences were found among groups for oxygen saturation, DLCO, acute exacerbations or death from any cause. BNP levels at week 24, however, showed a mean change from baseline of $-11.6 \mathrm{ng} \cdot \mathrm{L}^{-1}$ and $39.7 \mathrm{ng} \cdot \mathrm{L}^{-1}$ in the nintedanib-plus-sildenafil and the nintedanibplus-placebo groups, respectively (difference $\left.-51.3 \mathrm{ng} \cdot \mathrm{L}^{-1}, 95 \% \mathrm{Cl}-85.1--17.6\right)$.
Finally, no important differences regarding the occurrence of adverse events and severe adverse events were detected between treatment groups.

\section{Commentary}

Choosing a primary end-point in clinical trials for IPF treatment is a difficult and complex decision. So far, FVC is the measurement used in most phase III clinical trials for IPF as a surrogate primary endpoint, but its significance is still controversial [6, 7]. Nevertheless, both nintedanib and pirfenidone treatments were approved by the US Food and Drug Administration for IPF, as they showed a significant effect on FVC decline when compared to placebo [2, 8, 9]. IPF is a chronic and progressive disease that affects patients in almost every aspect of their lives, so prolonging life and slowing down FVC decline alone may not be enough to measure the impact of pharmacological treatment. The use of patient-reported outcome measures, such as disease-specific questionnaires, allows physicians to assess nonphysiological aspects of the patient and the disease [10]. In this regard, several tools have been validated for IPF and are of great use not only for clinical trials but also for routine clinical practice $[10,11]$. INSTAGE is the first treatment trial whose primary end-point was to improve QOL in IPF patients. This study used patient-related outcome measures to assess the impact of adding sildenafil to treatment with nintedanib, but it failed to show significant benefits regarding QOL measured by the SGRQ, UCSD-SOBQ or EQ-5D visual-analogue scale scores in this cohort of IPF patients with severe pulmonary gas exchange impairment. As stated by the investigators, it is possible that the study was underpowered, which could have affected the outcome, but other aspects may also contribute to obtaining these negative results. Both nintedanib and sildenafil treatments are frequently associated with side-effects that worsen patient QOL. Although no statistical differences were detected regarding occurrence of serious or severe adverse events between treatment groups, a higher occurrence of diarrhoea, nausea, vomiting, headaches, cough and dyspnoea was reported in the nintedanibplus-sildenafil group. Moreover, QOL is influenced by the presence of symptoms, exercise capacity, nutrition status or psychological burden, among others, but nintedanib and sildenafil have little effect in improving these aspects. Therefore, since no specific approach was used to address such issues, INSTAGE was less likely to find a significant QOL improvement.

This is one of the few clinical trials that includes IPF subjects with a DLCO $<35 \%$ of predicted, a subgroup of patients that has been poorly studied. A lower risk of FVC decline of $\geq 5$ percentage points of predicted value or death was observed in subjects treated with nintedanib plus sildenafil, although these results should be interpreted carefully as they 
differ with previous trials of endothelin receptor antagonist therapy in IPF [12, 13]. Nevertheless, changes in FVC and safety and side-effect profile in the nintedanib-plus-placebo group were similar to those observed in previous trials including patients with mild to moderate disease [2].

Finally, an absence of BNP level increase was observed in the nintedanib-plus-sildenafil group. This result, possibly related to the effect of sildenafil on vascular remodelling and right ventricular stress reduction $[14,15]$, may imply that the addition of sildenafil to antifibrotic therapy could have a larger impact in IPF patients with pulmonary hypertension and right ventricular dysfunction. Further trials are required and could be of great interest to assess this effect.

\section{Implications for practice}

Nintedanib is a safe and effective treatment for IPF patients. It can be considered for individuals with severe disease, as the safety profile and changes in FVC observed in the nintedanib-plus-placebo group were similar to those found in previous studies with mild-moderate IPF. The addition of sildenafil to nintedanib therapy does not improve QOL compared to nintedanib alone and is not recommended for IPF patients. However, it may have a beneficial effect in a specific subgroup of individuals with pulmonary hypertension and right ventricular dysfunction, but more studies are needed to confirm and quantify this potential effect.

\section{Affiliations}

\section{Guillermo Suarez-Cuartin ${ }^{1,2}$, Maria Molina-Molina ${ }^{1,2}$}

${ }^{1}$ Hospital Universitari de Bellvitge, L'Hospitalet de LLobregat, Spain. 'Institut d'Investigació Biomèdica de Bellvitge (IDIBELL), L'Hospitalet de LLobregat, Spain.

\section{Conflict of interest}

G. Suarez-Cuartin has nothing to disclose. M. Molina-Molina reports grants from Boehringer Ingelheim, Roche, GlazoSmithKline, Esteve-Teijin, Almirall and Chiesi outside the submitted work.

\section{References}

1. Raghu G, Remy-Jardin M, Myers JL, et al. Diagnosis of Idiopathic Pulmonary Fibrosis. An official ATS/ERS/JRS/ALAT clinical practice guideline. Am J Respir Crit Care Med 2018; 198: e44-e68.

2. Richeldi L, du Bois RM, Raghu G, et al. Efficacy and safety of nintedanib in idiopathic pulmonary fibrosis. N EnglJ Med 2014 370: 2071-2082

3. Idiopathic Pulmonary Fibrosis Clinical Research Network. A controlled trial of sildenafil in advanced idiopathic pulmonary fibrosis. N Engl J Med 2010; 363: 620-628.

4. Raghu G, Rochwerg B, Zhang Y, et al. An official ATS/ERS/ JRS/ALAT clinical practice guideline: treatment of idiopathic pulmonary fibrosis. An update of the 2011 clinical practice guideline. Am J Respir Crit Care Med 2015; 192: e3-e19.

5. Kolb M, Raghu G, Wells AU, et al. Nintedanib plus sildenafil in patients with idiopathic pulmonary fibrosis. N Engl J Med 2018; 379: 1722-1731.

6. Karimi-Shah BA, Chowdhury BA. Forced vital capacity in idiopathic pulmonary fibrosis - FDA review of pirfenidone and nintedanib. N EnglJ Med 2015; 372: 1189-1191.

7. Raghu G, Collard HR, Anstrom KJ, et al. Idiopathic pulmonary fibrosis: clinically meaningful primary endpoints in phase 3 clinical trials. Am J Respir Crit Care Med 2012; 185: 1044-1048.

8. Noble PW, Albera C, Bradford WZ, et al. Pirfenidone in patients with idiopathic pulmonary fibrosis (CAPACITY): two randomised trials. Lancet 2011; 377: 1760-1769.
9. King TE, Bradford WZ, Castro-Bernardini S, et al. A phase 3 trial of pirfenidone in patients with idiopathic pulmonary fibrosis. N EnglJ Med 2014; 370: 2083-2092.

10. Wijsenbeek $M$, van Manen M, Bonella F. New insights on patient-reported outcome measures in idiopathic pulmonary fibrosis. Curr Opin Pulm Med 2016; 22: 434-441.

11. Moor CC, Heukels P, Kool M, et al. Integrating patient perspectives into personalized medicine in idiopathic pulmonary fibrosis. Front Med 2017; 4: 226.

12. King TE, Behr J, Brown KK, et al. BUILD-1: a randomized placebo-controlled trial of bosentan in idiopathic pulmonary fibrosis. Am J Respir Crit Care Med 2008; 177: 75-81.

13. Raghu G, Million-Rousseau R, Morganti A, et al. Macitentan for the treatment of idiopathic pulmonary fibrosis: the randomised controlled MUSIC trial. Eur Respir J 2013; 42: 1622-1632.

14. Milara J, Escrivá J, Ortiz JL, et al. Vascular effects of sildenafil in patients with pulmonary fibrosis and pulmonary hypertension: an ex vivo/in vitro study. Eur Respir J 2016; 47: 1737-1749.

15. Han MK, Bach DS, Hagan PG, et al. Sildenafil preserves exercise capacity in patients with idiopathic pulmonary fibrosis and right-sided ventricular dysfunction. Chest 2013; 143 : 1699-1708. 